\title{
Delay-Constrained Admission and Bandwidth Allocation for Long-Reach EPON
}

\author{
Burak Kantarci, Hussein T. Mouftah \\ School of Electrical Engineering and Computer Science, University of Ottawa, Ontario, Canada \\ Email: $\{$ kantarci, mouftah\}@site.uottawa.ca
}

\begin{abstract}
Next generation Passive Optical Network (PON) technology has been evolving to consolidate the metro and access networks in order to offer enhanced capacity, high split ratio and reduced deployment cost per subscriber. However, transmission of the signals to long distances up to $100 \mathrm{~km}$ leads to increased propagation delay whereas high split ratio may lead to long cycle times resulting in large queue occupancies and long packet delays. In this article, we present a delay-constrained admission control mechanism and adapt this scheme to our previously proposed bandwidth allocation technique, namely Periodic GATE Optimization (PGO). We call this new scheme Delay-Constrained Periodic GATE Optimization (DC-PGO). DC-PGO is designed to run for multiple service classes as it inherits the advantages of PGO by periodically building and solving an ILP formulation at the OLT in order to obtain the appropriate credit values for the overloaded ONUs. At the ONU side, DCPGO runs an admission control scheme before pushing the arriving packets in the sub-queues. The admission control scheme uses statistical information consisting of the local data at the ONU and the previously received GATE messages from the OLT. Through simulations, we show that DC-PGO enhances the performance of multi-threaded polling in longreach Ethernet PON when packets of differentiated service classes arrive with pre-specified delay requirements.
\end{abstract}

Index Terms-Dynamic bandwidth allocation, Ethernet PON, long-reach passive optical networks, multi-threaded polling

\section{INTRODUCTION}

Optical Access Networks form a great portion of the telecommunication networks as they serve in the last mile of the Internet service provisioning. Passive Optical Networks (PONs) have emerged as a promising technology by offering high capacity and low power budget solutions [1]. Deployment of high capacity and low cost solutions in the last mile is required due to the increasing bandwidth demand and differentiated real-time service level requirements of the end user applications such as Internet Protocol (IP) telephony, video on-demand, online gaming and http [2]. ATM-PON (APON), Ethernet PON (EPON) and Gigabit PON (GPON) are current passive optical network technologies that are standardized in ITUT G.983, IEEE 802.3ah and ITU-T G.984, respectively. Basically a PON consists of an Optical Line Terminal (OLT) where the access network meets the metropolitan

This paper is an extended version of "On SLA-constraints in dynamic bandwidth allocation for long-reach passive optical networks," by B. Kantarci, and H. T. Mouftah, which appeared in the Proceedings of the $12^{t h}$ International Conference on Transparent Optical Networks (ICTON), Munich, Germany, June 2010. (c) 2010 IEEE.

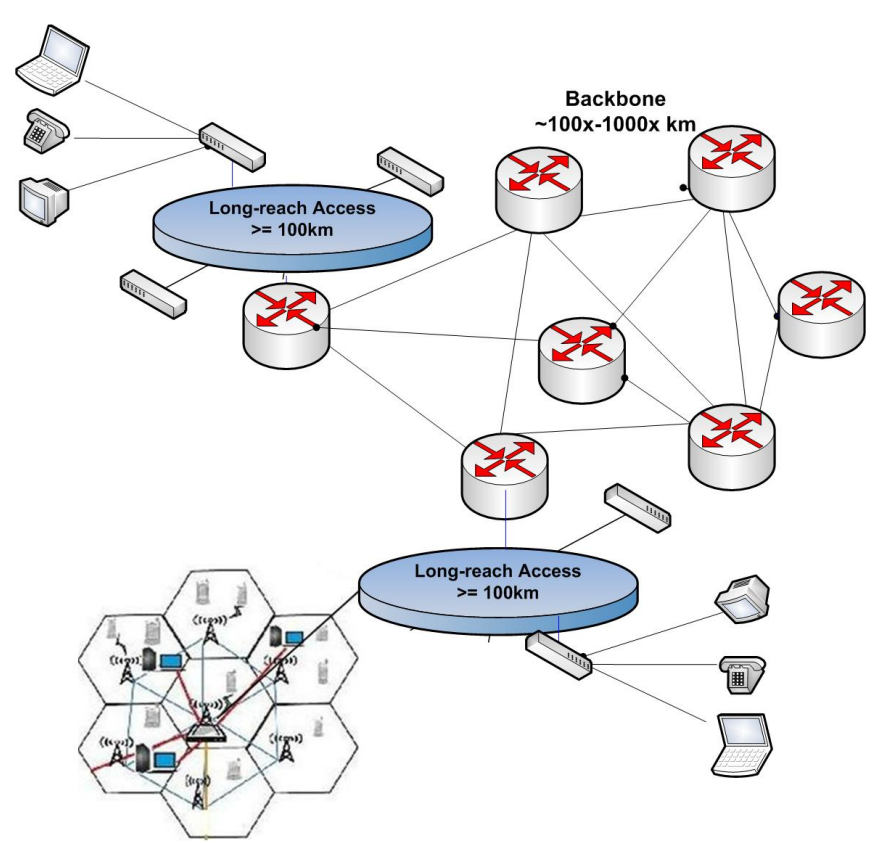

Figure 1. New infrastructure of the telecom network by the employment of LR-PON.

area network and several Optical Network Units (ONUs), and the end users are connected to the ONUs through wired (fiber, copper, etc) or wireless (WiFi, WIMAX, etc) links. A feeder fiber runs from the OLT to a passive splitter (coupler) which splits (couples) the signal on $N$ distribution fibers each of which is connected to an ONU.

Recently, Long-Reach PON (LR-PON) technology has been developed to combine the capacities of metro and access networks by extending the reach of the feeder and increasing the split ratio [3]. Conventional PON lies on a maximum feeder distance of $20 \mathrm{~km}$ while LR-PON aims to extend this distance to $100 \mathrm{~km}$ and even longer [4]-[7]. Figure 1 illustrates two LR-PONs having ring topologies of ONUs in a distance longer than $100 \mathrm{~km}$. As seen in the figure, the access network is not connected to a metro ring network but it is connected to the Internet directly through the edge routers of the backbone. Thus, by deployment of LR-PON, not only metro and access network capacities are combined but also the telecommunication network infrastructure is simplified [8].

Hardware demonstrations have already been introduced to implement the LR-PON technology [9], [10] however, long-reach and high split ratio of LR-PON introduces 
a significant drawback in terms of packet delay if conventional Multi Point Control Protocol (MPCP) signaling [11] and bandwidth distribution schemes [12]-[14] are employed. Those existing solutions have initially been proposed for the conventional feeder distances (up to $20 \mathrm{~km}$ ) which are considerably shorter than the aimed distance of LR-PONs. As it is stated in [15], [16], round trip time of a single frame increases up to $1 \mathrm{~ms}$ in an extended reach of $100 \mathrm{~km}$, and considering the REPORTGATE messaging of the MPCP, the ONU is expected to introduce further delay to an arriving user frame since the arriving frame will be granted after sending a REPORT message from the ONU which will be followed by a GATE message from the OLT.

There are several other types of long-reach optical access networks such as long-reach GPON [17], long-reach DWDM PON [18], hybrid long-reach TDM/DWDM PON [3], and radio over fiber through long-reach PON [19]. However, the scope of this article is limited to LongReach EPON (LR-EPON).

In this paper, we present an admission control mechanism which is adapted from our previously proposed scheme, Periodic GATE Optimization with Quality of Service (QoS)-Awareness (PGO-QoS) [20], [21] where the packets arrive at the ONUs with pre-specified delay bounds. Our new scheme is called Delay-Constrained Periodic GATE Optimization (DC-PGO), and it has initially been proposed in [22]. Here, we present this scheme in more details and evaluate its delay, utilization, packet loss and queue length performance by running extensive simulations. In PGO-QoS, the OLT polls the ONU requests in a multi-threaded way while periodically running an optimization model that re-adjusts the credits for the overloaded ONUs in order to minimize the average packet delay. The ONUs run a burstification (intra-ONU scheduling) module in order to prioritize the de-queuing procedure of the high-priority SLA sub-queues and aid the optimization building process of the OLT. DC-PGO employs an admission control module at each ONU in front of the burstification module, and based on the SLA subqueue lengths and thread durations, it estimates whether an arriving packet can be dequeued and transmitted to the OLT within its specified delay bounds. The simulation results show that combining a delay prediction-based admission control and PGO-QoS achieve keeping the delay of multi-threaded polling low for high priority classes as well as the overall performance. Furthermore, DC-PGO does not lead to a reduction in the utilization of the LREPON. We also adopt the estimation function in the multithreaded MPCP, compare the performance enhancements obtained by delay-awareness, and show that employment of DC-PGO in LR-EPON is advantageous in terms of packet delay, packet loss ratio, channel utilization and total queue length.

The paper is organized as follows. In Section II, we briefly summarize the related work on DBA in LR-EPON. In Section III, we revisit our previously proposed provisioning framework, PGO-QoS that forms a basis to our

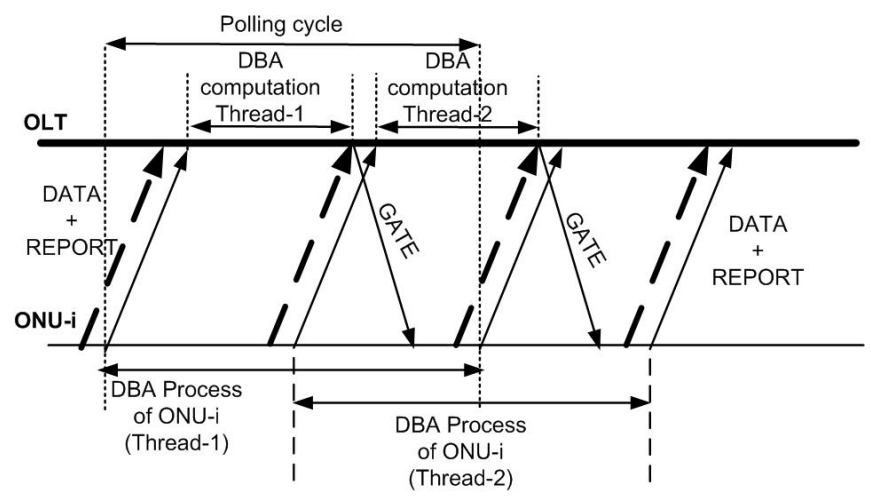

Figure 2. Polling ONUs with two threads [24].

proposal, and we present our proposed delay constrained enhancement, DC-PGO, in detail. Section IV presents numerical results and related discussions. We conclude our paper and give future directions in Section V.

\section{RELATED WORK}

In the literature, several solutions have been proposed to overcome the performance degradation problem in LR-EPON. A comprehensive survey of the bandwidth distribution solutions in LR-PONs can be found in [23]. Song et al. have proposed multi-threaded polling in order to reduce the gaps in the upstream channel due to exchanging REPORT and GATE messages between the ONUs and the OLTs [15], [16]. Multi-threaded polling is built on top of the conventional MPCP-based interleaved polling however, in multi-threaded polling, the OLT polls the ONU requests through multiple threads. Thus, an ONU (say ONU- $i$ ) does not have to wait the GATE $\left(G A T E_{i}^{k}\right)$ message of the last REPORT message $\left(R E P_{i}^{k}\right)$ to send a request for an incoming user packet since a GATE message that belongs to another polling thread $\left(G A T E_{i}^{k+1}\right)$ will arrive before $G A T E_{i}^{k}$. Hence, the request can be sent through the REPORT message of the $(k+1)^{t h}$ thread $\left(R E P_{i}^{k+1}\right)$. Figure 2 illustrates a scenario of one complete polling cycle which was presented in [24]. In the figure, the OLT polls the ONUs by two parallel threads. The whole polling cycle emulates two interleaved bandwidth distribution processes leading to two short sub-cycles of the main polling cycle. In [15], [16], it is shown that by the employment of multi-threaded polling, average packet delay of the limited DBA in LREPON can be reduced significantly until the load gets heavier to almost full utilization of the upstream feeder channel. Skubic et al. further enhanced the multi-threaded polling by proposing a coordination scheme between the multiple polling threads [24] so that over-granting/lategranting the ONUs is avoided and uplink utilization is improved as well.

In [25], the authors propose a bandwidth allocation scheme for LR-EPON where at the beginning of each granting cycle, the OLT determines the idle upstream timeslots in the next cycle and credits one or both of the ONUs whose REPORT messages are causing the 
gap. In [26], an adaptive polling scheme is proposed for LR-EPON in order to differentiate various classes of service packets. This scheme is further improved to utilize the upstream gaps through inserted polling cycles [26]. In [27], a fair online distribution of the excessive bandwidth at each polling cycle is introduced in order to overcome the instability of the bandwidth distribution beyond certain critical loads. In [28], the authors have proposed a GATE-Driven bandwidth distribution scheme where the OLT uses the information collected from the REPORT messages and determines when to send the next GATE message. As stated in [29], this scheme converges to a multi-server polling system in long-term. In [30], we have proposed an adaptive threshold-based reporting scheme on top of the multi-threaded polling to reduce average packet delay. In [21], [31], we have proposed an enhancement to the multi-threaded polling, namely Periodic GATE Optimization (PGO) and its Quality of Service (QoS)-aware adaptation PGO-QoS [20]. PGO adopts multi-threaded polling, and it periodically formulates an integer linear programming (ILP) model in order to determine how to credit the overloaded ONUs at each thread until the next formulation period.

\section{Delay-Constrained Admission CONTROL AND DBA IN LONG-REACH EPON}

The proposed mechanism is mainly based on our previously proposed scheme, Periodic GATE Optimization with Quality of Service Awareness (PGO-QoS). Hence, in this section, we start with a brief description of PGO-QoS, and then, we proceed with our proposed delay-constrained admission control scheme, Delay-Constrained Periodic GATE Optimization (DC-PGO). For detailed explanation and performance evaluation of PGO-QoS, the reader is referred to [21].

\section{A. Periodic GATE Optimization (PGO) Revisited}

In [21], we have proposed Periodic GATE Optimization (PGO) and its quality of service (QoS)-aware adaptation (PGO-QoS) as an enhancement to multi-threaded polling. PGO-QoS consists of two modules, namely the burstification module running at the ONUs and the bandwidth distribution module running at the OLT.

Burstification module (i.e., intra-ONU scheduling) determines the de-queuing proportion for the SLA queues (sub-queues) at each ONU. For a scenario with three SLA classes, the packets are expected to be dequeued with respect to the dequeuing proportion of $r_{1}: r_{2}: r_{3}$ where $r_{c}$ denotes the component of the proportion for the SLA queue-c, and priority of SLA class- $c$ is greater than the priority of SLA class- $d$ when $c>d$. Burstification module at the ONU periodically updates the dequeuing proportion components, and $\sum_{c} r_{c}=R$ where $R \geq 10$. ONU periodically monitors the sub-queue lengths. De-queuing proportion update starts by checking the highest priority queue length, and if the queue length have been increasing, the corresponding component in the de-queuing proportion is marked to be incremented. In this case, in order to keep the sum of the dequeuing components $(R)$ constant, it attempts to decrement the de-queueing component of a lower priority class. Increment/decrement continues with the lower priority class components. The details of this procedure can be found in [21]. Once, the de-queuing proportions are obtained, each ONU produces a polynomial value as shown in Eq. 1 where $X$ is an arbitrarily large integer. The polynomial value increases dramatically with longer high-priority sub-queues while low-priority sub-queues slightly increase the polynomial value. In other words, an ONU which produces a larger polynomial value is supposed to have more high priority packets to be transmitted in comparison to an ONU with a smaller polynomial value. Each ONU appends this polynomial value to the REPORT message at the end of each update period so that the OLT knows the class-based distribution of the packets at the corresponding ONU.

$$
P_{i}(X)=\sum_{c=0}^{C-1} X^{c} r_{c}^{i}
$$

Bandwidth distribution module of PGO-QoS is almost the same as PGO itself in [31] with a slight difference by taking the polynomial into account. The OLT periodically builds an Integer Linear Programming (ILP) formulation in order to determine the appropriate credits for the overloaded ONUs during the next period at each polling thread. Overloaded ONU refers to an ONU whose last request at thread $k\left(R_{i}^{k}\right)$ exceeds the minimum guaranteed bandwidth (maximum allowed timeslot), i.e., $R_{i}^{k}>W_{\max }$. Last bandwidth demands received through each thread are used as the input of the formulation. The objective of the ILP formulation is to minimize the optimized grant of each ONU at each thread as seen in Eq. 2 where $\Lambda_{i}^{k}$ stands for the optimized grant of ONU- $i$ at thread $k$. Optimized grant denotes the granted bandwidth to an overloaded ONU by satisfying the fairness among the SLA classes as well as the polling sub-cycles.

$$
\begin{array}{r}
\text { minimize } \sum_{k} \sum_{i} \Lambda_{i}^{k} \\
R_{\text {excess }}^{k}=\sum_{i \in Q}^{N}\left(W_{\max }-R_{i}^{k}\right)
\end{array}
$$

The optimization model formulates the following major constraints: $i$ ) The excessive bandwidth at thread $k$ ( $R_{\text {excess }}^{k}$ as shown in Eq. 3), ii) Limiting the total allocated bandwidth for the overloaded ONUs $(Q$ : Set of overloaded ONUs) and the lightly loaded ONUs ( $L$ : Set of lightly loaded ONUs) above by the maximum cycle byte time $\left(T_{\max }\right)$, iii) Setting the minimum guaranteed bandwidth to the ONUs to the maximum allowed timeslot, and differentiation between each peer of an ONU pair with respect to the last requests and the polynomial values reported by them. Formulation of the constraints is straightforward except the one given in Eq. 4 hence, here we only explain this constraint. According to the equation, 
the OLT aims to issue more grants for the ONUs that have longer queue lengths and more number of high priority packets.

$$
\Lambda_{i}^{k}-\frac{R_{i}^{k} \cdot P_{i}(X)}{R_{j}^{k} \cdot P_{j}(X)} \cdot \Lambda_{j_{j \neq i}}^{k} \geq 0, \quad \forall k \wedge R_{i}^{k}<R_{j}^{k}
$$

In Eq. 5, $\Lambda_{i}^{k}$ is the optimized GATE value of ONU-i for thread $k$ at the last optimization solution, $P_{i}(X)$ denotes the polynomial value sent by ONU- $i$, and $R_{i}^{k}$ stands for the bandwidth demand of ONU- $i$ at thread $k$. Whenever an ONU is overloaded, until the next ILP formulation period, it is granted by using the formulation in Eq. 5 where $Q$ denotes the set of overloaded ONUs. Thus, the ONU is granted the maximum allowed bandwidth and a portion of the total excessive bandwidth which is proportional with its calculated credit value for the corresponding thread $k$.

$$
G_{i \epsilon Q}{ }^{(k)}=\left\{W_{M a x}+\sum_{j \in Q} R_{j_{\text {excess }}}^{k} \cdot \frac{\left(\Lambda_{i}^{k}-W_{\text {Max }}\right)}{\sum_{j \in Q}\left(\Lambda_{j}^{k}-W_{M a x}\right)}\right\}
$$

\section{B. Delay Constrained Periodic GATE Optimization (DC- $P G O)$}

PGO-QoS was designed to work with multiple SLA queues however, since packets may arrive with prespecified delay bounds, it calls for a control scheme in order to admit or drop the user packets upon arrival. Since the upstream channel is a contention domain, the delay experienced by an arriving packet can be affected by the dynamic profile of the incoming traffic of the corresponding ONU as well as the other ONUs. Thus, some of the packets are likely to be marked as lost at the destination since they are not able to arrive within the required delay bounds. In order to utilize bandwidth more efficiently, ONUs call for an admission control scheme which returns the estimated delay of an arriving packet and determines to drop/admit the packet based on the delay estimation. We add this mechanism to PGO-QoS and name it Delay-Constrained Periodic GATE Optimization (DC-PGO). Fig. 3 illustrates the block diagram of an ONU running the burstification and admission control schemes of the proposed framework.

In DC-PGO, the admission control unit of an ONU uses the sum of the sub-queue lengths $\left(Q_{c}\right)$, maximum allowed bandwidth $\left(W_{\max }\right)$ and the time difference between two successive threads $\left(\Delta t_{R E P}\right)$ in order to formulate the estimated delay $\left(D_{\text {est }}\right)$. As seen in Eq. 6, admission control module of DC-PGO assumes that the corresponding ONU will be granted $W_{\max }$ until the packet is dequeued. By dividing the total sub-queue lengths by the maximum allowed timeslot, it estimates an approximative number of sub-cycles to wait. Then, by simply multiplying the estimated number of sub-cycles with the time difference between the last two REPORT message departures, estimated delay $\left(D_{\text {est }}\right)$ of an arriving packet is obtained.

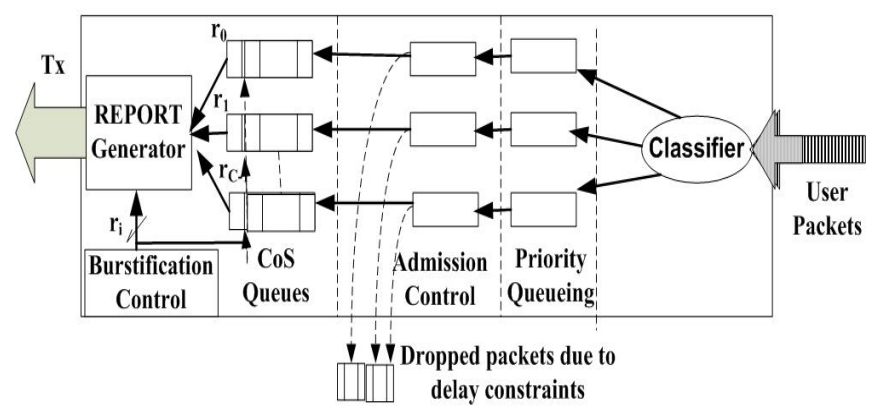

Figure 3. ONU block diagram for DC-PGO.

An average grant of $W_{\max }$ seems to be a naïve assumption since whenever the ONU is lightly loaded $\left(\sum_{k} Q_{c} \leq\right.$ $\left.W_{\max }\right)$, the $\mathrm{ONU}$ is granted the requested bandwidth to transmit $\sum_{k} Q_{c}$ bytes. However, the DBA module of DC-PGO credits the overloaded ONUs based on the results of the periodically run ILP formulation. As stated in the previous subsection, the enhancement introduced by the DBA module of DC-PGO (i.e., PGO-QoS) is to determine a heterogeneous distribution of the excessive bandwidth among the ONUs. Thus, $\Lambda_{i}^{k}$ denoting the bandwidth grant for ONU- $i$ at thread $k$, the DBA module already guarantees $\Lambda \geq W_{\max }$ for the overloaded ONUs. Hence, considering the dynamic profile of the incoming traffic, the assumption of an average grant of $W_{\max }$ is reasonable. It is worth to note that the success of the delay estimation process can be enhanced by the employment of further efficient methods such as data mining, pattern recognition or linear regression techniques.

As seen in Algorithm 1, each arriving packet is classified with respect to the SLA specifications that also include the delay requirements of its corresponding SLA class $c\left(\Theta^{c}\right)$. The estimated delay $\left(D_{\text {est }}\right)$ for the arriving packet is compared to the delay bound of its SLA class $\left(\Theta^{c}\right)$. If $D_{e s t} \leq \theta^{c}$, the packet is admitted and enqueued in the appropriate $\operatorname{CoS}$ queue (i.e., sub-queue), and its bandwidth request is issued by the next REPORT message. Otherwise, the packet is not admitted to its $\operatorname{CoS}$ queue and dropped since it is assumed to be possibly marked as a lost packet at the destination due to violation of the delay bound.

$$
D_{\text {est }}=\left(\left\lfloor\frac{\sum_{k} Q_{c}}{W_{\max }}\right\rfloor+1\right) \cdot \Delta_{R E P}
$$

Consolidation of PGO-QoS and the admission control scheme defined above is expected to enhance the delay performance of the LR-PON for the following reasons. DC-PGO aims to keep the SLA queues available for the arriving packets. Hence, packet loss is mostly due to the violation of the delay bounds although the system experiences packet losses due to buffer overflow as well. Packet delay is a function of the queueing, granting and dequeuing delays. Employment of a look-ahead mechanism to admit the incoming packets is expected to result with shorter buffers which lead to shorter queueing and dequeuing delays. Besides, DBA module of DC- 


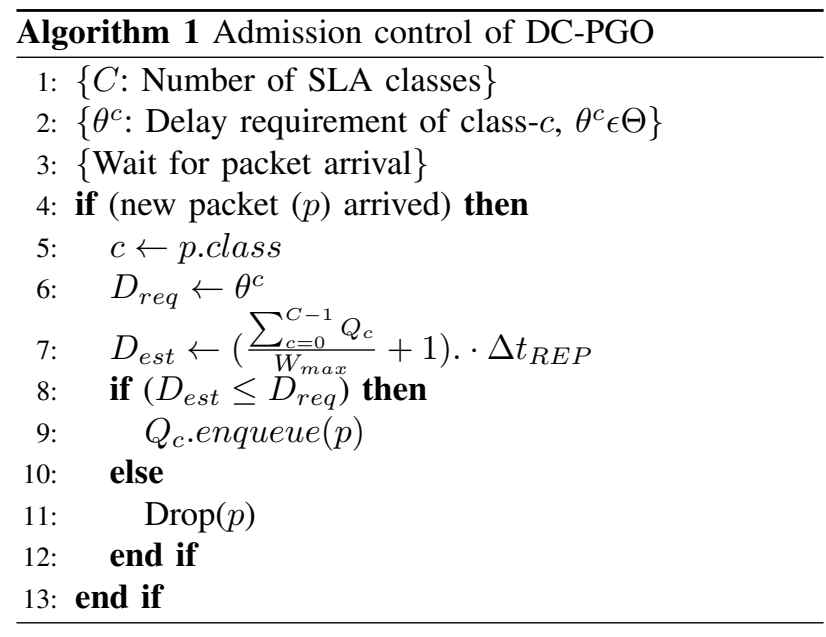

PGO aims to reduce the granting delays for all packets. Moreover, co-operation of the burstification and the DBA modules further reduces the delay for the high priority classes. Furthermore, due to the same reasons, DC-PGO introduces improved transparency to the changes in the SLA scenarios as the network load gets heavier as we present in the next section.

\section{NUMERICAL RESULTS}

Performance evaluation of DC-PGO is done by discrete event simulation implemented in Visual $\mathrm{C}++$ while the periodic optimization results of the DBA module are provided by CPLEX. The LR-EPON consists of 16 ONUs that are connected to the OLT via feeder fiber of $100 \mathrm{~km}$. User traffic capacity is assumed to be $100 \mathrm{Mbps}$ while the fiber link is assumed to offer 1 Gbps capacity. ONUs are assumed to have total buffer capacities of $1 \mathrm{MB}$. Incoming packets size is assumed to be distributed between 64B and 1500B. To be coherent with the previous work [21], OLT builds the ILP formulation upon receiving every $10^{4}$ packets. Number of SLA classes $(C)$ is assumed to be three where the priority order is as follows: $S L A_{\text {class }_{1}}<$ $S L A_{\text {class }_{2}}<S L A_{\text {class }_{3}}$. In order to be coherent with the realistic scenarios, user packets are distributed with respect to the proportion of $2: 1: 1$. Thus, the cheapest class has the highest density. Initial thread cycle is assumed to be $0.3 \mathrm{~ms}$ while the OLT polls the ONUs by three threads as in [16], [21]. DC-PGO runs multi-threaded polling as a basis, and in order to present its enhancement to multi-threaded polling (MT), we also adapt the admission control mechanism into MT.

Two different delay requirement sets $(\Theta)$ are assumed and tested under DC-PGO where $\Theta=\left\{\theta^{1}, \theta^{2}, \theta^{3}\right\}$.

Delay Set-1: $\Theta=\{150 \mathrm{~ms}, 75 \mathrm{~ms}, 5 \mathrm{~ms}\}$

Delay Set-2: $\Theta=\{120 \mathrm{~ms}, 50 \mathrm{~ms}, 3 \mathrm{~ms}\}$

We initially, evaluate the delay performance of DCPGO as illustrated in Fig. 4. As seen in the figure, under both scenarios, combining the admission control scheme at the tail of the input queues and PGO-QoS in the burstification and the DBA modules enhances the overall packet delay performance of multi-threaded polling.

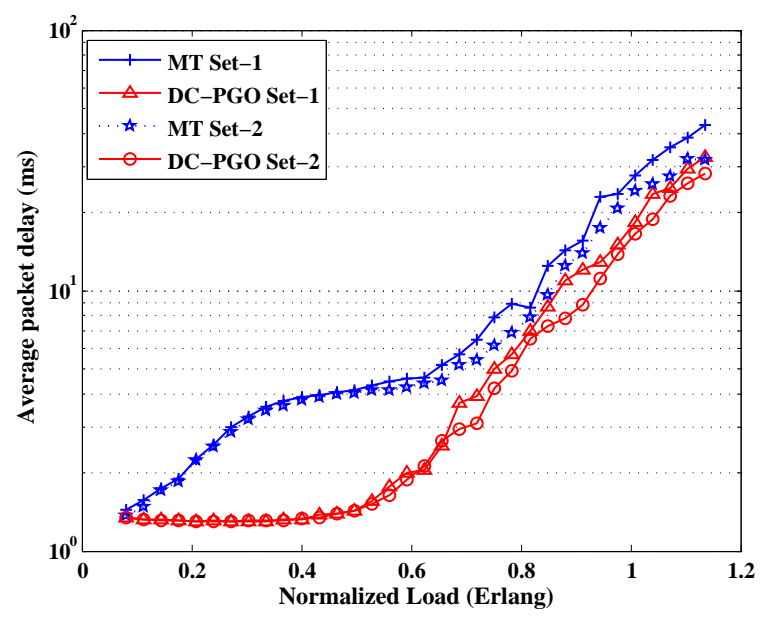

Figure 4. Average packet delay enhancement by DC-PGO under both delay constraint sets.

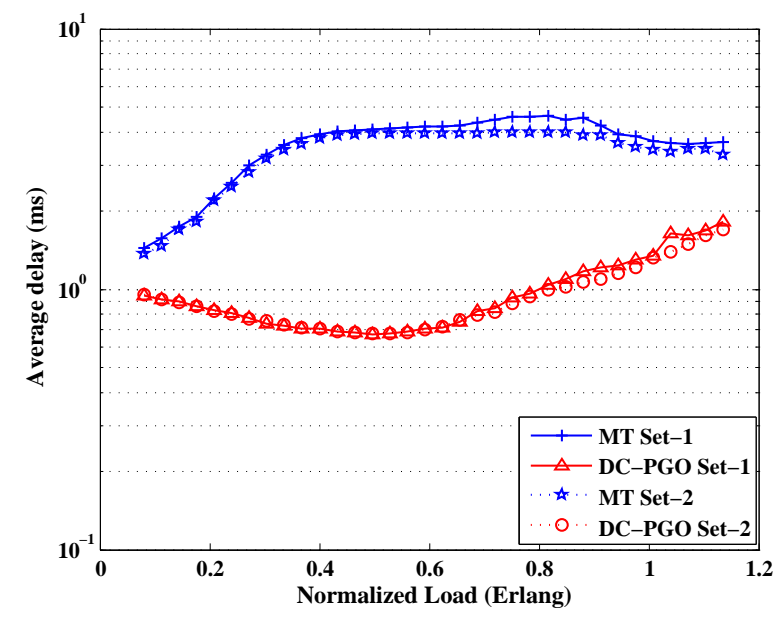

Figure 5. Average packet delay enhancement for SLA class-3 by DCPGO under both delay constraint sets.

Besides, employment of the second scenario, leads to reduction for both MT and DC-PGO under moderate and heavy loads since arriving packets have lower bounds under the second scenario. This phenomenon can also be seen in Fig.5-7 where the average delays for the packets of $\mathrm{SLA}_{\text {class }_{3}}-\mathrm{SLA}_{\text {class }_{1}}$ are represented, respectively.

Fig.5 illustrates the delay performance of DC-PGO and MT under both scenarios for the packets of $\mathrm{SLA}_{\text {class }_{3}}$. For each scenario, DC-PGO enhances the performance of MT for the packets of this class. Beyond the moderate loads, setting lower delay bounds for the arriving packets helps reducing the average delay for $\mathrm{SLA}_{\text {class }_{3}}$ under both schemes. Hence, the admission control scheme adopted by MT and DC-PGO ensures delay-awareness. Fig. 6 illustrates the same performance evaluation results for the packets of $\mathrm{SLA}_{\text {class }_{2}}$.

Since burstification and DBA modules of DC-PGO prioritize $\mathrm{SLA}_{\text {class }_{3}}$ and $\mathrm{SLA}_{\text {class }}$, starting from the moderate loads, it introduces longer delays to the packets of $\mathrm{SLA}_{\text {class }_{1}}$ when compared to MT as seen in Fig. 7. 


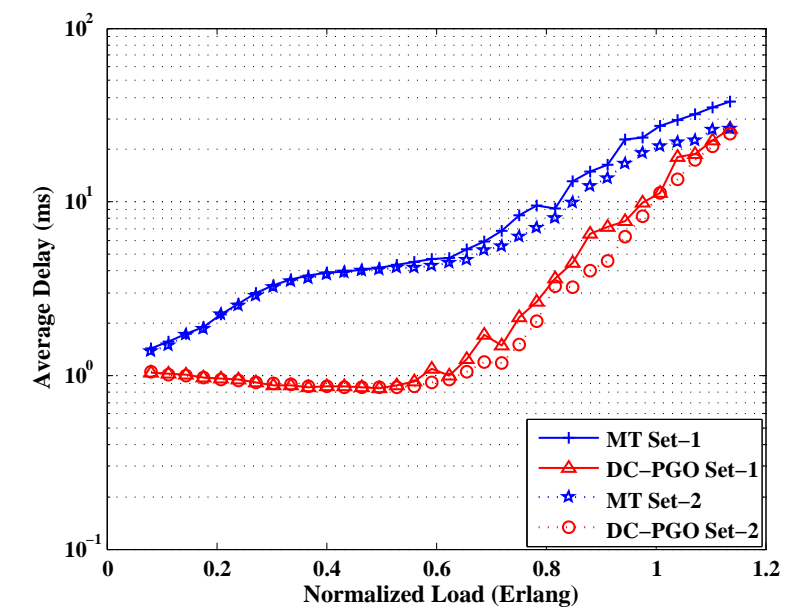

Figure 6. Average packet delay enhancement for SLA class-2 by DCPGO under both delay constraint sets.

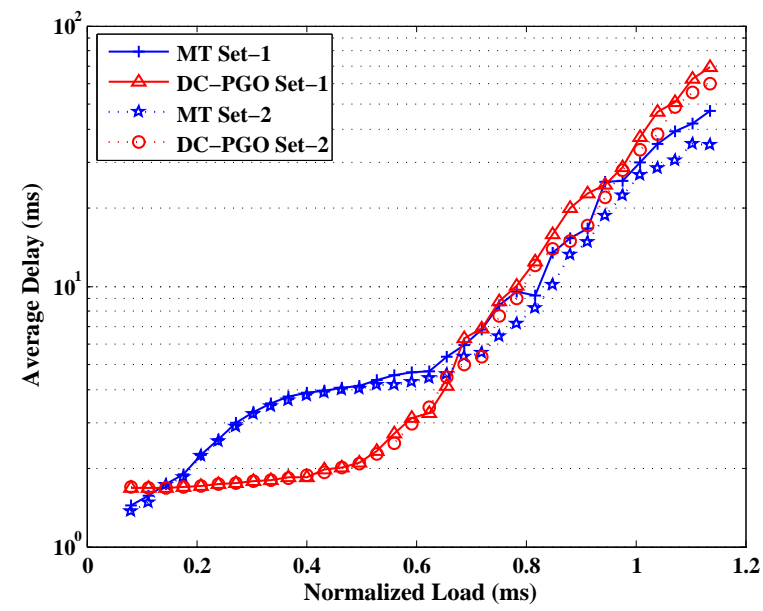

Figure 7. Average packet delay for SLA class-1 under DC-PGO and MT.

However, in reality, it is reasonable for DC-PGO (and for any differentiated provisioning scheme) to treat the low-priority packets according to the best effort service strategy. Hence, the behavior in the figure does not mask the advantageous aspects of DC-PGO.

In Fig. 8, average packet drop probability under DCPGO and MT is presented for both scenarios. The results in the figure are consistent with those in the previous figures. First, in the second scenario, both schemes drop more packets when compared to their performance in the first scenario. Since the delay bounds are lower in DelaySet-2, packets are dropped earlier due to delay bound violation which results with increased packet delay. Beyond 1.0 Erlang, the network gets heavily loaded hence, other than the delay bound violation, buffer overflow arises as another reason for packet losses. Hence packet loss performances of DC-PGO and MT coincide under heavy loads. On the other hand, DC-PGO leads to lower packet delay until the network load of 1.0 Erlang in both scenarios. The reason for dropping less packets

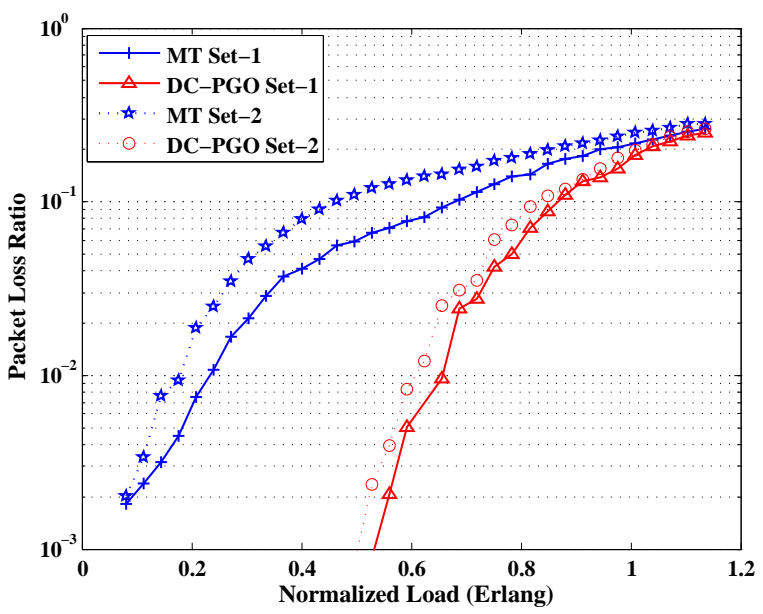

Figure 8. Average packet drop ratio under DC-PGO and MT.

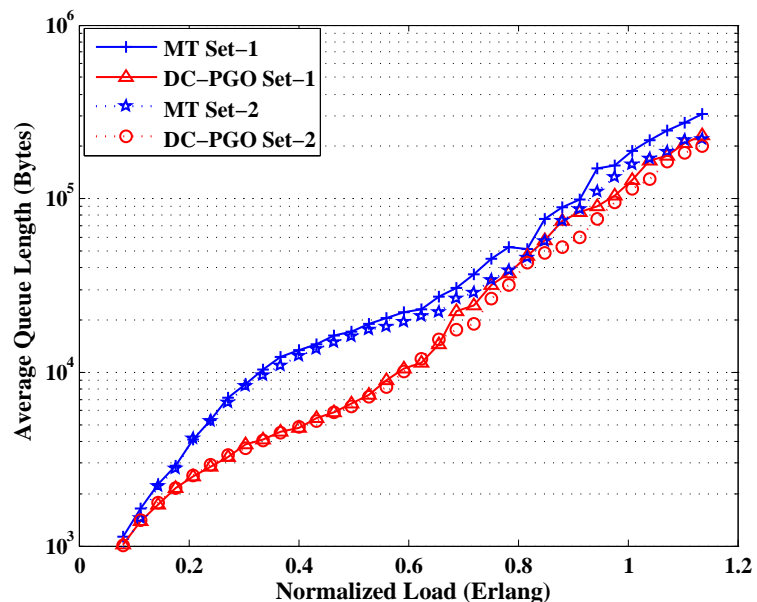

Figure 9. Average queue length under DC-PGO and MT.

in DC-PGO is two-fold: First, burstification and DBA modules of DC-PGO leads to reduced overall packet delay as we have seen in Fig. 4. Hence, when compared to MT with limited DBA, DC-PGO is more likely to meet the delay specifications. Thus, introducing reduced overall delay is one of the factors which also reduces the packet drop probability. The second factor affecting the packet loss probability is the total buffer occupancy. Periodically optimized crediting mechanism at the OLT assists the overloaded ONUs to burst their buffers in order to minimize the granting delays which results in reduced occupancies at the input buffers. Fig. 9 illustrates the total queue length behavior under DC-PGO and MT for both delay set scenarios. In both scenarios, DC-PGO succeeds in keeping the queue length below MT due to the reasons mentioned above. Thus, reduced buffer occupancy arises as the second factor for reduced packet loss probability.

Fig.10 compares the channel utilization performance of DC-PGO to that of MT under the two delay sets. As seen in the figure, DC-PGO is not affected significantly by the change in the contract scenario (delay requirement set) in 


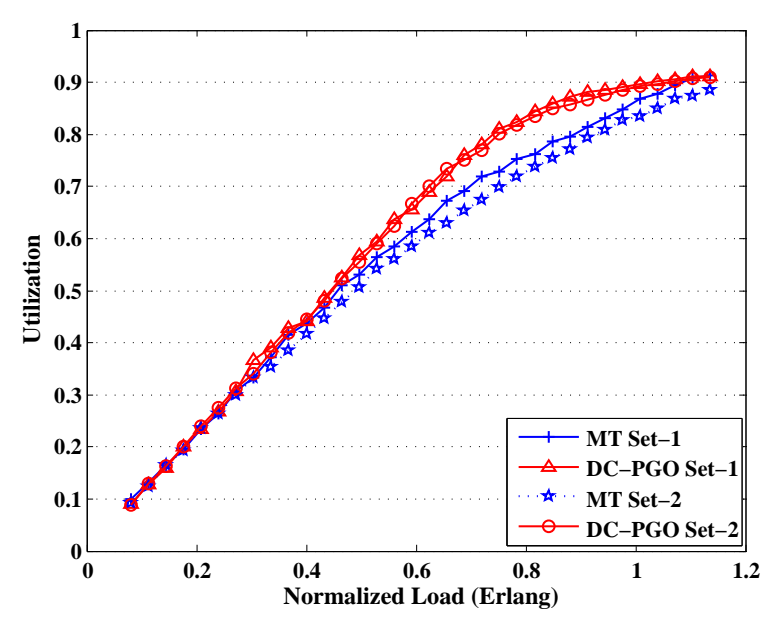

Figure 10. Average channel utilization under DC-PGO and MT.

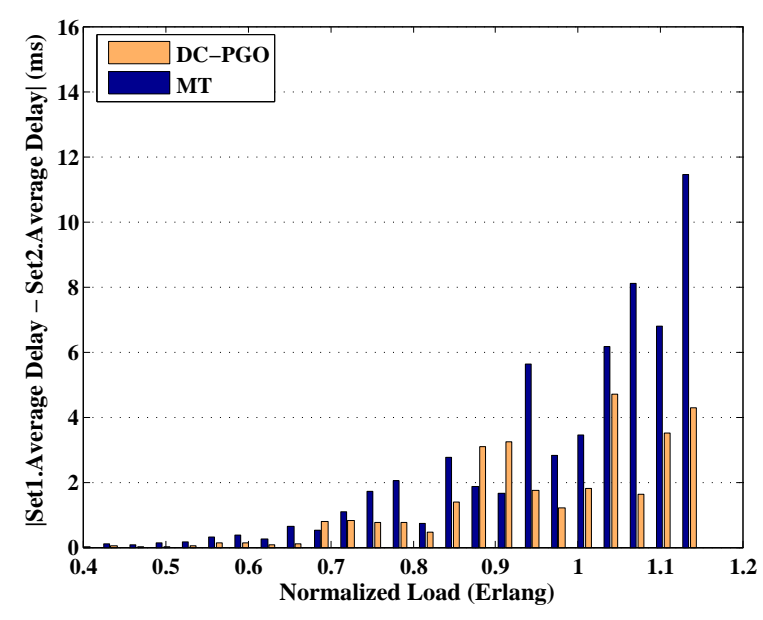

Figure 11. Transparency of DC-PGO to the change in the contract scenario.

terms of utilization while MT leads to reduced utilization under Delay-Set-2 scenario where packet delay requirements of the three SLA classes are lower than those under the Delay-Set-1 scenario. As we have already shown in Fig. 8, DC-PGO does not show a significant change in packet loss probability performance since at the OLT, it aims to adapt the credits of the overloaded ONUs in order to minimize the granting delay. Hence, channel utilization of DC-PGO seems to be more transparent to the change in the contract scenario. The second phenomenon is that DC-PGO does not lead to a reduced utilization while reducing the average packet delay. On the contrary, under each scenario, starting from 0.4 Erlang until 1.0 Erlang network load, it leads to enhanced utilization for both delay requirement scenarios.

We have clearly seen that DC-PGO demonstrates transparency in terms of overall delay and utilization performance when the delay requirement scenario changes in Fig. 4 and Fig. 10. In Fig. 11, by presenting statistical data, we illustrate the transparency of DC-PGO and MT to the change in the delay requirement scenario. The $y$-axis in the figure denotes the absolute difference between the average delay per packet under Delay-Set-1 and DelaySet-2 scenarios. As seen in the figure, MT without PGOQoS leads to a maximum average delay difference of $11.5 \mathrm{~ms}$ while DC-PGO introduces a maximum average delay difference of $4.5 \mathrm{~ms}$.

\section{CONCLusion}

Long-Reach Ethernet PON (LR-EPON) suffers from long packet delays due to increased feeder distance hence, new solutions have been proposed in the literature to replace the conventional bandwidth distribution mechanisms. In this article, we have presented a modified version of our previously proposed bandwidth distribution scheme, namely Periodic GATE Optimization with Quality of Service Awareness (PGO-QoS). PGO-QoS has two modules, namely the dynamic bandwidth allocation (DBA) module running at the OLT in order to minimize the granting delay per ONU and the burstification module running at each ONU to differentiate de-queuing process at the SLA sub-queues. We have called the proposed modified version Delay-Constrained Periodic GATE Optimization (DC-PGO). DC-PGO runs in an environment where the packets arriving at an ONU belong to differentiated Service Level Agreement (SLA) classes. Thus, each arriving packet has a specific delay requirement. Hence, in DC-PGO, we have presented an admission control scheme that runs for each arriving packet. The packets are enqueued if they are forecasted to arrive at the OLT within their delay bounds. Otherwise, they are dropped in order to reserve space for the arriving packets in the related sub-queues. We have also modified the multi-threaded (MT) polling which forms a basis for DCPGO as well as its successor PGO-QoS so that MT runs the same admission control for the arriving packets with differentiated delay requirements. We have evaluated the performance of DC-PGO in comparison to that of MT by employing two delay requirement set scenarios for three SLA classes. Through simulations, we have shown that DC-PGO introduces reduced average delay per packet as well as reduced average delay for the packets of the high priority service classes. Furthermore, simulation results verify that DC-PGO leads to reduced packet loss and consequently better utilization until the heavy network loads. The simulation results further show that DC-PGO is more transparent to the change in delay requirement set as the average delay and utilization performances are less affected by the change in the packet delay bounds.

Currently, we are extending this study by adapting DCPGO into survivable LR-EPON architectures to guarantee both enhanced delay performance and reliability.

\section{REFERENCES}

[1] J. Zheng and H. T. Mouftah, Optical WDM Networks: Concepts and Design Principles. Wiley-IEEE Press, Hoboken, NJ, 2004. 
[2] H. Naser and H. T. Mouftah, Broadband Access Networks, Chapter 7: Dynamic Bandwidth Allocation for Ethernet Passive Optical Networks. Shami A., Maier M., Assi C., Eds.; Springer, 2009, pp. 151-167.

[3] D. Shea and J. Mitchell, "Long-reach optical access technologies," IEEE Network, vol. 21, no. 5, pp. 5-11, 2007.

[4] R. Davey, D. Nesset, A. Rafel, D. Payne, and A. Hill, "Designing long reach optical access networks," BT Technology Journal, vol. 24, pp. 13-19, 2006.

[5] K. Ennser, M. Zannin, and S. Taccheo, "Extending reach of passive optical networks through optical amplification," in International Conference on Transparent Optical Networks,(ICTON), 28 2009-July 2 2009, pp. 1-4.

[6] L. Gutierrez, M. de andrate, and S. Sallent, "New trends on optical access networks: DBAs for 10G EPON and LongReach PON," in European Conference on Networks and Optical Communications, June 2009, pp. 487-494.

[7] H. Song, B.-W. Kim, and B. Mukherjee, "Long-reach optical access," in Broadband Access Networks, A. Shami, M. Maier, and C. Assi, Eds., 2009.

[8] _ _ , "Long-reach optical access networks: A survey of research challenges, demonstrations, and bandwidth assignment mechanisms," IEEE Communications Surveys \& Tutorials, vol. 12 issue 1, pp. 112-123, First Quarter 2010.

[9] R. P. Davey, D. B. Grossman, M. Rasztovits-Wiech, and D. B. Payne, "Long-reach passive optical networks," IEEE/OSA Journal of Lightwave Technology, vol. 27 no 3, pp. 273-291, February 2009.

[10] D. P. Shea and J. E. Mitchell, "A 10-Gb/s 1024-way-split 100-km long-reach optical-access network," IEEE/ OSA Journal of Lightwave Technology, vol. 25, no. 3, pp. 685693, Mar. 2007.

[11] "IEEE 802.3ah - Ethernet in the First Mile working group archives," [Online] http://www.ieee802.org/3/efm.

[12] G. Kramer, B. Mukherjee, and G. Pesavento, "IPACT: A dynamic protocol for an ethernet PON (EPON)," IEEE Communications Magazine, vol. 40 (2), pp. 74-80, 2002.

[13] M. Mcgarry, M. Reisslein, and M. Maier, "Ethernet passive optical network architectures and dynamic bandwidth allocation algorithms," IEEE Communications Surveys Tutorials, vol. 10, no. 3, pp. 46-60, 2008.

[14] J. Zheng and H. T. Mouftah, "A survey of dynamic bandwidth allocation algorithms for ethernet passive optical networks," Optical Switching and Networking, vol. 6 issue 3, pp. 151-162, July 2009.

[15] H. Song, A. Banerjee, B.-W. Kim, and B. Mukherjee, "Multi-Thread Polling: A Dynamic Bandwidth Distribution Scheme in Long-Reach PON," in IEEE Global Telecommunications Conference, 2007, 2007, pp. 24502454.

[16] H. Song, B.-W. Kim, and B. Mukherjee, "Multi-thread polling: A dynamic bandwidth distribution scheme in longreach PON," IEEE Journal on Selected Areas in Communications, vol. 27 issue 2, pp. 134-142, February 2009.

[17] K. Kanonakis and I. Tomkos, "Offset-based scheduling with flexible intervals for evolving GPON networks," IEEE/OSA Journal of Lightwave Technology, vol. 27 no 15, pp. 3259-3268, August 2009.

[18] D. Shea and J. Mitchell, "Architecture to integrate multiple pons with long reach dwdm backhaul," Selected Areas in Communications, IEEE Journal on, vol. 27, no. 2, pp. 126 $-133,2009$.

[19] K. Prince, A. V. Osadchiy, and I. T. Monroy, "WiMAX Radio-on-Fibre in $118-\mathrm{km}$ Long-Reach PON with Deployed Fibre," in 35th European Conference on Optical Communication (ECOC), Sep. 2009.

[20] B. Kantarci and H. Mouftah, "Periodic GATE Optimization with QoS-awareness for Long-Reach Passive Optical Networks," in IEEE Symposium on Computers and Communications (ISCC), 2010, pp. 879-884.
[21] B. Kantarci and H. T. Mouftah, "Periodic GATE optimization (PGO): A new service scheme for long-reach passive optical networks," IEEE Systems Journal, vol. 4/4, pp. 440-448, Dec. 2010.

[22] — , "On SLA-constraints in dynamic bandwidth allocation for long-reach passive optical networks," in International Conference on Transparent Optical Networks (ICTON), June 2010, Tu.B1.3.1 - Tu.B1.3.7.

[23] — , "Bandwidth distribution solutions for performance enhancement in long-reach passive optical networks," Communications Surveys Tutorials, IEEE, vol. PP, no. 99, pp. $1-20,2011$.

[24] B. Skubic, J. Chen, J. Ahmed, B. Chen, L. Wosinska, and B. Mukherjee, "Dynamic bandwidth allocation for long-reach PON: overcoming performance degradation," Communications Magazine, IEEE, vol. 48, no. 11, pp. 100-108, Nov. 2010.

[25] N. Merayo, T. Jimenez, P. Fernandez, R. Duran, R. M. Lorenzo, I. deMiguel, and E. Abril, "A bandwidth assignment polling algorithm to enhance the efficiency in QoS long-reach EPONs," European Transactions on Telecommunications, [Online] http://dx.doi.org/10.1002/ett.1449, November 2010.

[26] N. Merayo, J. T, R. J. Duran, d. M. I. Fernandez, P., R. M. Lorenzo, and E. J. Abril, "Adaptive polling algorithm to provide subscriber and service differentiation in a Long-Reach EPON," Photonic Network Communications, vol. 19, pp. 257-264, 2010.

[27] J. R. Ferguson, M. Reisslein, and M. McGarry, "Online excess bandwidth distribution for Ethernet passive optical networks," Journal of Optical Networking, vol. 8, pp. 358369, April 2009.

[28] N. Antunes, C. Fricker, P. Robert, and J. Roberts, "GATEDriven Dynamic Wavelength and Bandwidth Allocation for WDM-EPONs," in IEEE Global Telecommunications Conference, 2010, pp. 1-5.

[29] - "Upstream traffic capacity of a WDM EPON under online GATE-driven scheduling, internal report, 2010."

[30] B. Kantarci and H. T. Mouftah, "Bandwidth Distribution with Adaptive Threshold-based Optical Burst Assembly in Long-Reach EPON," in OSA Advanced Photonics Congress, 2011.

[31] B. Kantarci and H. Mouftah, "Periodic GATE Optimization (PGO) in Long-Reach Passive Optical Networks," in 25th Queen's Biennial Symposium on Communications (QBSC), May 2010, pp. 230-233.

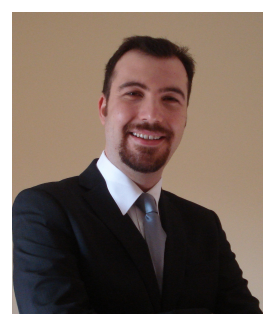

Burak Kantarci is currently a postdoctoral fellow at the School of Electrical Engineering and Computer Science of the University of Ottawa. He received the M.Sc, and Ph.D degrees in Computer Engineering at Istanbul Technical University (ITU) in 2005 and 2009, respectively. He received the Siemens Excellence Award in 2005 for his studies in optical burst switching. During his Ph.D study, he was a visiting scholar under the supervision of Prof. Mouftah at the University of Ottawa (20072008). He has co-authored over four dozen refereed papers in established journals and conferences, and contributed three book chapters. His research interests are energy-efficient network planning, broadband access networks, WDM networks, optical switching, survivable network design, green communications and cloud computing. 


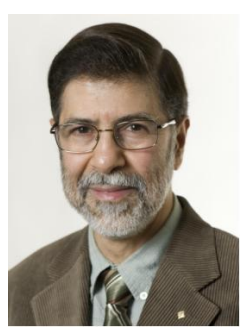

Hussein T. Mouftah joined the School of Information Technology and Engineering (SITE) of the University of Ottawa in 2002 as a Tier 1 Canada Research Chair Professor, where he became a University Distinguished Professor in 2006. He has been with the ECE Dept. at Queen's University (1979-2002), where he was prior to his departure a Full Professor and the Department Associate Head. He has six years of industrial experience mainly at Bell Northern Research of Ottawa (became Nortel Networks). He served IEEE ComSoc as Editor-in-Chief of the IEEE Communications Magazine (1995-97), Director of Magazines (199899), Chair of the Awards Committee (2002-03), Director of Education (2006-07), and Member of the Board of Governors (1997-99 and 200607). He has been a Distinguished Speaker of the IEEE Communications Society (2000-07). Currently he serves IEEE Canada (Region 7) as Chair of the Awards and Recognition Committee. He is the author or coauthor of 7 books, 49 book chapters and more than 1000 technical papers, 12 patents and 140 industrial reports. He is the joint holder of 12 Best Paper and/or Outstanding Paper Awards. He has received numerous prestigious awards, such as the 2008 ORION Leadership Award of Merit, the 2007 Royal Society of Canada Thomas W. Eadie Medal, the 2007-2008 University of Ottawa Award for Excellence in Research, the 2006 IEEE Canada McNaughton Gold Medal, the 2006 EIC Julian Smith Medal, the 2004 IEEE ComSoc Edwin Howard Armstrong Achievement Award, the 2004 George S. Glinski Award for Excellence in Research of the U of O Faculty of Engineering, the 1989 Engineering Medal for Research and Development of the Association of Professional Engineers of Ontario (PEO), and the Ontario Distinguished Researcher Award of the Ontario Innovation Trust. Dr. Mouftah is a Fellow of the IEEE (1990), the Canadian Academy of Engineering (2003), the Engineering Institute of Canada (2005) and the Royal Society of Canada RSC: The Academy of Science (2008) 